\title{
Temporally Asymmetric Fluctuations are \\ Sufficient for the Operation of a Correlation
}

\author{
Ratchet \\ Dante R. Chialvo 2 and Mark M. Millonas ${ }^{1,2}$ \\ ${ }^{1}$ Complex Systems Group, Theoretical Division, and Center for Nonlinear Studies, \\ MS B258, Los Alamos National Laboratory, Los Alamos, NM 87545, USA. \\ ${ }^{2}$ Fluctuations and Biophysics Group, Santa Fe Institute, \\ 1660 Old Pecos Trail, Suite A, Santa Fe, NM 87501, U.S.A.
}

May 7, 2019

\begin{abstract}
A number of recent attempts to understand broad principles of energy transduction in biological systems have focused on correlation ratchets - systems which extract work out of fluctuations which are correlated in time.[1, 2, 3, 4, 5, 6] Correlation ratchets are "information engines" analogous to Maxwell's Demon, which extract work out of a bath by using information about the system to "choose" only
\end{abstract}


those fluctuations which are helpful to make the engine run. [8] This information, which can only be acquired if the Demon is not in equilibrium with the bath, [9] can be used to rectify the energy already available, but otherwise inaccessible in the thermal bath. Processes like this, in which the energy stored in a nonequilibrium bath is transformed into work at the expense of increased entropy, are believed to be the basis of "molecular motors", and are of great importance in biology, and a number of other fields. It has been shown that the combination of a broken spatial symmetry in the potential (or ratchet potential) and time correlations in the driving are crucial, and enough to allow the transformation of the fluctuations into work. 1] The required broken spatial symmetry implies a specific molecular arrangement of the proteins involved. Here we show that a broken spatial symmetry is not required, and that temporally asymmetric fluctuations (with mean zero) can be used to do work, even when the ratchet potential is completely symmetric. Temporal asymmetry, defined as a lack of invariance of the statistical properties under the operation to temporal inversion, is a generic property of nonequilibrium fluctuation, and should therefore be expected to be quite common in biological systems. 
In biological systems energy stored in the form of ATP molecules is used to drive various biochemical processes. Since new ATP molecules are constantly being made, and the degraded ones removed from the system, the bath of energy molecules is very far from equilibrium. The question of how this tremendous negative entropy allows for an effective transduction of energy at the nanoscale in a very noisy environment is greatly complicated by the fact that there are no principles of the power and generality of those of equilibrium statistical mechanics for nonequilibrium systems.

It has been found that, under the right conditions spatially homogenous fluctuations with mean zero can be used to do work if they are correlated in time. [1, 2, 3, 田司, 6] These systems we call “correlation ratchets". [7] These time correlation are due to the coupling of a system to a bath which is not in equilibrium, and patently do not violate the second law of thermodynamics. These systems are indeed analogs of Maxwell's Demon, and operate as information engines. As shown by Szilard [10], the information is acquired at the expense of an entropy increase of the Demon, an observation which salvages the second law. Similarly it has been shown that correlation ratchets works at the expense of the total increase of entropy of the bath, and run off of the physical information (negentropy) contained in the nonequilibrium bath. [8]

Here we consider the simplest imaginable system which contains the crucial elements. We strongly emphasize here that it is not the model, which is indeed trivial, or its simplifying assumptions, but the physical principal of tem- 
poral asymmetry we illustrate which is important. This physical principle has not been discussed before to the best of our knowledge in any context, and certainly not with respect to correlation ratchets and biological energy transduction. Although the specific model has been chosen as a simple illustration, understandable to a broad audience, the behavior which it exhibits is quite general indeed, and we hope extension to more complicated and realistic situation will present themselves naturally to the mind of any attentive reader who grasps our main point.

We consider a particle in the piecewise linear potential pictured in Fig. 1(a), as considered in [1]. The potential is periodic and extends to infinity in both directions. $\lambda$ measures the spacing of the wells, $\lambda_{1}$ and $\lambda_{2}$ the inverse steepnesses of the potential in opposite directions out of the wells, and $Q$ the well depths. The particle undergoes overdamped Brownian motion due to its coupling with a thermal bath of temperature $T$, and external driving $F(t)$ with represent the nonequilibrium forces. The expression for the current in the adiabatic limit, which measures the work done by the ratchet has already been derived in this case, 11, 11] where

$$
\begin{gathered}
J(F)=\frac{P_{2}^{2} \sinh (\lambda F / 2 k T)}{k T(\lambda / Q)^{2} P_{3}-(\lambda / Q) P_{1} P_{2} \sinh (\lambda F / 2 k T)} \\
P_{1}=\delta+\frac{\lambda^{2}-\delta^{2}}{4} \frac{F}{Q}, \quad P_{2}=\left[1-\frac{\delta F}{2 Q}\right]^{2}-\left(\frac{\lambda F}{2 Q}\right)^{2} \\
P_{3}=\cosh [(Q-\delta F / 2) / k T]-\cosh (\lambda F / 2 k T)
\end{gathered}
$$

where $\lambda=\lambda_{1}+\lambda_{2}$ and $\delta=\lambda_{1}-\lambda_{2}$. The average current, the quantity of 
primary interest, is given by

$$
<J>=\frac{1}{\tau} \int_{0}^{\tau} J(F(t)) d t
$$

where $\tau$ is the period of the driving force $F(t)$, which is assumed longer than any other time scale of the system in this adiabatic limit. Magnasco considered this case, but only for $F(t)$ symmetric in time $F(t)=F(n \tau-t)$. Here we will again consider a driving with a zero mean, $\langle F(t)>=0$, but which is asymmetric in time,

$$
F(t)= \begin{cases}\left(\frac{1+\epsilon}{1-\epsilon}\right) A & 0 \leq t<\frac{\tau}{2}(1-\epsilon), \quad \bmod \tau \\ -A & \frac{\tau}{2}(1-\epsilon)>t \leq \tau, \quad \bmod \tau\end{cases}
$$

as shown in Fig. 1(b). In this case the time averaged current is easily calculated,

$$
<J>=\frac{1}{2}[(1+\epsilon) J(A)+(1-\epsilon) J(-(1+\epsilon) A /(1-\epsilon))] .
$$

Fig. 2 shows that the current is a peaked function of the amplitude of the driving. Thus, everything else being constant, there is an optimal amplitude for the driving. Similarly Fig. 3 shows that the current is also a peaked function of $k T$. The driving, the potential, and the thermal noise in fact play cooperative roles. Unless $A$ is quite large, there are no transition out of the wells when $k T=0$, and therefore no current, but if the noise is too large it washes out both the potential and the details of the driving, and the current again goes to zero. Similarly, without the driving the transitions in either direction are the same, but if the driving is too large the potential plays less of a selective role, and the current drops back down. Here the main features introduced by 
the temporal asymmetry are the interplay of the lower potential barriers in the positive direction relative to the negative direction (for this particular driving) and the corresponding shorter and longer times respectively the force is felt. These types of competitive effects appear ubiquitously in systems where there is an interplay between thermal activation and dynamics, including, but by no means limited to, the phenomenon of stochastic resonance.

Figs. 2 and 3 are for completely symmetric potentials. In these cases the exponential Arrhennius dependence of the thermal activations over the barriers overcomes the time factors, and the current is in the opposite direction of that which is produced by a temporal symmetry and a spatial asymmetry. This effect is of course reversed if $\epsilon \rightarrow-\epsilon$. There can also be competitive effects between the temporal asymmetry and the spatial asymmetry, as pictured in Fig. 4, which gives rise to a very unusual switching of the direction of the current as the asymmetry factor $\epsilon$ is varied. This reversal represent the competition of the spatial asymmetry, which dominates for small $\epsilon$ an the temporal asymmetry, which dominates for large $\epsilon$.

Temporal asymmetry is definitely not related to the simplified adiabatic approximation presented above, and is just as valid for fast noise. For instance, the general explanation of the phenomena shown in Figs. 1-3 given above is equally applicable to the case where the period of the driving is large with respect to the intra-well relaxation time, but not large with respect to the firstpassage times over the barriers, such it as the case of stochastic resonance. 12 
Additional argument can be made for even faster noise.

In cannot be emphasized enough that the reasons for the phenomena we discuss here are not related to the specific approximations which allowed for an analysis solution in our very simple illustration, but are ubiquitous characteristics of nonequilibrium fluctuations, and deserve to be studied in more detail. The main idea here, that of a temporal asymmetry in the driving, can be easily incorporated by extending the theory of Ref. [2] to continuous, but non-Gaussian noise, 13] and the discrete noise models of Ref. [5], with qualita-

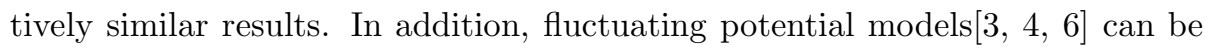
made to in corporate temporal asymmetry as an important factor if the potential changes shape as it fluctuated in magnitude. A detail example of this type of system is also forthcoming in a separate publication. 14]

Our main point here, which should be clearly understood, is that temporal asymmetry, defined as a lack of invariance of the statistical properties of a system under the operation of time inversion, can be expected to be ubiquitous property of most nonequilibrium systems, and can give rise to nonequilibrium transport, such as that which is utilized by many biological processes. We believe it is a fundamental principle, equal in importance to Magnasco's recent observation that spatial asymmetry and temporal correlations are sufficient for nonequilibrium transport. [1]

This principle has never been pointed out before, even though it might easily have been demonstrated by means of rather simple extensions of the models of 
Ref. [1, 5] if the respective authors had realized the general significance of this type of nonequilibrium asymmetry. The authors of Ref. [5] came close to this point when they pointed out that an spatially asymmetric marginal density of the dichotomous fluctuations they use would give rise to a current. In their specific context this might under certain conditions be regarded as a type of temporal asymmetry, but they neither analyze this case, nor draw any distinction between this situation and the case where there is a net force. More importantly, the more general conclusions we draw here cannot be inferred from this comment, which only applies to their specific model. They do not point out, as we do here for the first time, that temporal asymmetry is the crucial property, and the this property is a quite generic aspect of nonequilibrium fluctuation, and not a property of a specific model.

Time correlations in the driving will influence the transitions in either direction. Since this influence depends on the shape of the potential, as well as the properties of the noise, an asymmetry in the potential will give rise to a net current. However, when the noise is temporally asymmetric, its correlation properties in either direction are different, and a net current can arise even in the absence of a spatial asymmetry. Note that the dependence of the strength and direction of the current on the properties of the noise discussed here is not related to the similar results of Refs. [2] and [5], in which only temporally symmetric fluctuations where considered, and the current vanished in the spatially symmetric case. 
Such temporal asymmetry is present in virtually any waveform or noise with a nontrivial distribution of frequency component phases. It is thus interesting to note that this possibility has not been considered explicitly in any previous study of correlation ratchets. It is believed that many driven biochemical processes work by cycling though a sequence of intermediary states. These cycles are driven by the steps in the hydrolysis of ATP. If the steps in this sequence have different time scales, which is generally the case, the result will be temporal asymmetry, as well as correlations. Unless there is a physical (generally equilibrium) restriction, temporal asymmetry will probably be ubiquitous in nonequilibrium systems, such as biological energy transducers.

It is exciting to note that now that the motion of individual motor proteins can be tracked at the level of single steps 15, 16, it may be possible to test some of these ideas via an analysis of the time series of the motion of the molecule. Temporal asymmetry will appear as the nonvanishing of the odd

moments (of any order other than first, e.g. the bicovariance $<F(t) F^{2}(s)>$ ) of the fluctuations, an observation which may be of some use to experimentalists.

\section{References}

[1] M. Magnasco, Phys. Rev. Lett. 71, 1477 (1993).

[2] M. M. Millonas and D. I. Dykman, Phys. Lett. A 183, 65 (1994).

[3] D. Astumian and M. Bier, Phys. Rev. Lett. 72, 1766 (1994) 
[4] J. Prost, J.-F. Chauwin, L. Peliti, and A. Ajdari, Phys. Rev. Lett. 72, 2652 $(1994)$

[5] C. Doering, W. Horsthemke and J. Riordan, Phys. Rev. Lett. 72, 2984 (1994).

[6] G. Oster, C. Peskin, and G. B. Ermentrout (to be published).

[7] This term appears to have been introduced by George Oster in regards to fluctuating potential models, but here we extend this terminology to cover fluctuating force modes as well.

[8] M. M. Millonas, submitted to Physical Review Letters.

[9] L. Brillouin, J. Appl. Phys. 22, 334 (1951).

[10] L. Szilard, Z. Phys 53, 840 (1929).

[11] The simple illustration of Magnasco, and the one used here, can be derived using straightforward techniques found earlier in H. Risken, The FokkerPlanck Equation, Springer-Verlag (2nd Ed.) (1984).

[12] It should be noted here that for the same reason the basic phenomena described in Magnasco's illustration (Ref. [1]) will also be more or less unchanged in this faster noise case. Something which seems to have escaped a number of authors.

[13] D. Chialvo and M. M. Millonas (submitted to Phys. Rev. Lett.). 
[14] T. Elston and M. M. Millonas (in preparation).

[15] K. Svoboda, C. F. Schmidt, B. J. Schnapp and S. M. Block, Nature 365, 721 (1993).

[16] K. Svoboda, and S. M. Block, preprint.

ACKNOWLEDGMENTS: Work partially supported by NIMH. The Santa Fe Institute receive funding from the John D. Catherine T. MacArthur Foundation, NSF, and the U.S. Department of Energy. 
Figure 1: (a) Plot of the simple piecewise ratchet potential, where the spatial degree of asymmetry is given by the parameter $\delta=\lambda_{1}-\lambda_{2}$. (b) Plot of the driving force $F(t)$ which preserved zero mean $\langle F(t)\rangle=0$, where the temporal asymmetry is given by the parameter $\epsilon$. 
Figure 2: Plot of the current vs. $A$ for a symmetric potential $(\delta=0)$, with $Q=1, \lambda=1, \epsilon=1 / 2$ and (a) $k T=.3$, (b) $k T=.1$, (c) $k T=.01$. 
Figure 3: Plot of the current vs. $k T$ for a symmetric potential $(\delta=0)$, with $Q=1, \lambda=1, \epsilon=1 / 2$ and (a) $A=1$, (b) $A=.8$, (c) $A=.5$. 
Figure 4: Plot of the current vs. the time asymetry parameter $\epsilon$ with $Q=1, \lambda=$ $1, k T=.01$, and $A=2.1$, for (a) a symetric potential $\delta=0$ and asymmetric potentials (b) $\delta=-.3$ (c) $\delta=-.7$ 